\title{
Desarrollo y gestión de carreras con adultos en el siglo XXI: lecturas hacia una armonización de lo global y de lo individual
}

\author{
Manuel Joaquim Enriques Rafael
}

Facultad de Psicología y de Ciencias de la Educación, Universidad de Lisboa

\section{Portugal}

$\underline{\text { mrafael@fpce.ul.pt }}$ 


\section{Resumen}

La historia de la Orientación Profesional ha producido un amplio y riquísimo conjunto de teorizaciones y de intervenciones y un volumen substancial de literatura. Muchas de las contribuciones surgieron como consecuencia de respuestas a necesidades de la sociedad, desde las primeras concepciones de Parsons, pasando por las propuestas del desarrollo de la carrera a lo largo de la vida, hasta las actuales teorías contextuales y constructivistas. A pesar de la multitud de contribuciones, la población adulta ha merecido particular atención con posterioridad, para lo que ha contribuido un conjunto de factores de naturaleza demográfica y tecnológica. Algunos de esos factores son presentados en este artículo, así como los aspectos más relevantes hacia la comprensión y la intervención con adultos proporcionados por diferentes teorías y autores sobre el desarrollo de la carrera. Considerando que, en la actualidad y, en particular, en lo que se refiere al mundo del adulto, la expresión Orientación es menos ajustada, se discuten, también, en este artículo, los desafios que se plantean al Desarrollo y Gestión de Carreras con adultos, surgidos de las evoluciones de la sociedad en el comienzo del siglo XXI, en parte consecuencia del fenómeno de la globalización. Se discuten, de este modo, algunas respuestas del Desarrollo y Gestión de Carreras en los niveles Global / Internacional, Organizacional e Individual.

Palabras Clave: orientación; desarrollo y gestión de carreras; adultos; global; organizacional; individual 


\section{Introducción: primeras lecturas}

El Desarrollo y Gestión de Carreras en adultos es un área relativamente nueva. Mientras la Orientación, en una perspectiva científica, se ha iniciado hace cerca de 100 años con Frank Parsons, la formulación de modelos abarcando el ciclo vital y la atención, en particular, a las especificidades de los sujetos adultos, son preocupaciones bastante más recientes.

Las teorías y las intervenciones en el contexto de la Orientación estuvieron durante largos años exclusivamente dedicadas al adolescente. Ilustrativa de esta situación es la siguiente afirmación «al mirar un texto típico de psicología, cualquier persona seria inducida a pensar que el desarrollo humano se completa a los 21 años» (Hopson y Scally, 1980). La evolución de estas concepciones y, en particular, el surgimiento de modelos del desarrollo a lo largo de la vida, así como un conjunto de factores de naturaleza demográfica y tecnológica han hecho despertar en la sociedad, en general, y en la comunidad científica, en particular, un interés por el estudio del adulto. Se constata, por ejemplo, que los cambios en la vida del sujeto adulto son un fenómeno cada vez más frecuente debido en parte a las incertidumbres del ambiente económico y de los cambios tecnológicos, bien como al surgimiento de nuevas actitudes individuales hacia el trabajo.

Ha empezado, en consecuencia, a surgir un número creciente de investigaciones sobre la carrera y el ajuste vocacional de adultos de todas las edades de modo que, en los últimos cuarenta años, ha ocurrido un profundo cambio en el contexto del estudio del desarrollo de la carrera manifiesto en el surgimiento de trabajos con muestras de profesionales y adultos que compiten en número con las que consideran adolescentes y estudiantes universitarios.

Considerando el dominio del adulto, como un tema fundamental en el Desarrollo y Gestión de Carreras, este artículo tiene como objetivos generales: a) presentar algunos de los factores que conducen al incremento del interés de la Orientación hacia los adultos, después de una preocupación teórica y de intervención inicialmente dedicada solamente a los jóvenes; b) analizar, de diferentes autores (y perspectivas), contribuciones para la comprensión, teorización e intervención con adultos y c) reflejar los principales desafíos, necesidades y oportunidades que se plantean, en la actualidad, al Desarrollo y Gestión de Carreras con adultos. 


\section{Una lectura de la historia: de la adolescencia a la pertinencia del estudio del adulto}

El surgimiento de la Psicología Vocacional como problema científico ha tenido lugar a principios del siglo XX, subrayado por un enfoque cuya preocupación fundamental ha consistido en la comparación entre las características del individuo y las demandas de las profesiones en el momento en el que éste ingresaba en el mundo del trabajo. Este enfoque, apellidado de rasgo-factor, debió su éxito, en grande medida, a Frank Parsons, cuyas ideas se han formalizado en la obra titulada Choosing a Vocation.

La teorización de Parsons es, en sí misma, de simple elaboración; utilizando las palabras del autor "la orientación (profesional) consiste en tres pasos: el primero, una clara comprensión de si mismo, de sus aptitudes, capacidades, recursos, limitaciones (...) y otras cualidades; segundo, un conocimiento del mundo del trabajo: sus exigencias y condicionantes; ventajas e inconvenientes, oportunidades (...) tercero, una combinación de las relaciones entre estos dos grupos de factores" (Parsons, 1909, p.5). Esta teorización llevará a profundos y largos reflejos en la teoría y en la práctica de la Orientación hasta la década de los 50 del siglo XX (Crites, 1969; Hackett, Lent y Greenhaus, 1991) siendo particularmente significantes, en la Península Ibérica, los trabajos de los españoles Mira y López y José Germain y los del portugués Faria de Vasconcelos. Binet, Piéron, Munsterberg y Claparéde constituyen, igualmente, figuras de renombre, en este capítulo, en Europa (Ferreira Marques, 1993; Vasconcelos, 1936).

Progresivamente se reconoce que el proceso de Orientación es algo más complejo que la descripción hecha por Parsons, constituyendo etapas de particular importancia, en las décadas de los 30 hasta los 50, por una parte, la evolución de la psicología diferencial y de la psicometría (con el surgimiento de los tests colectivos de evaluación de las aptitudes, del primer inventario de Strong y con el estudio científico de las profesiones) y, por otra, la publicación, en 1942, de la obra Counseling and Psychoterapy de Rogers; esta obra, aunque haya planteado el enfoque en los aspectos psicoterapéuticos, tuvo particular influencia en la concepción da la orientación centrada en el individuo, aspecto manifiesto en la importancia de los aspectos motivacionales y afectivos, en el enfoque en la autoaceptación y en la autocomprensión, en una atención a las verbalizaciones del sujeto como fuente de información y, además, en el estímulo a la investigación y práctica sobre la interacción cliente y consejero (Crites, 1969, 1981). 
Un cambio radical en las concepciones teóricas en Orientación surge en la década de los 50 del siglo XX con la emergencia de las teorías del desarrollo de la carrera a lo largo de la vida. El carácter innovador de estas teorías consiste en el entendimiento del desarrollo psicológico humano según una perspectiva de ciclo vital, atribuyendo particular relieve al estudio de todos los cambios en el contexto del desarrollo individual físico, intelectual, afectivo, social, de la personalidad y vocacional (Jepsen, 1990). El trabajo de Ginsberg y colaboradores constituye un aporte decisivo para este cambio (Ginzberg, 1952) por la razón de estimular el incremento de las perspectivas del desarrollo aunque limitado por restringirse al período de la adolescencia y del comienzo de la edad adulta.

Las primeras formulaciones teóricas de Super contemplando todo el ciclo vital constituyen, indubitablemente, en este contexto, un nuevo y decisivo impulso (Super, 1953, 1957). Investigaciones con un evidente caracter longitudinal tales como el Career Pattern Study de Super, el Career Development Study, de Bribbons y Lohnes y el Project Talent de Flanagan han proporcionado, a su vez, bases empíricas importantes (Ferreira Marques, 1993).

De este modo, en la década de los 60, las designaciones Carrera y Desarrollo de la Carrera se han popularizado. La creciente importancia de las perspectivas del desarrollo constituye, también, una tendencia en la década de los 70, a lo largo de la cual han surgido aportes tan importantes como son la autonomía de la Psicología Vocacional entendida como el estudio del comportamiento y del desarrollo vocacionales (Crites, 1969) y el surgimiento del movimiento de la educación de la carrera estableciendo la unión de la orientación a la educación, al curriculum escolar y al proceso educativo (Herr y Cramer, 1996).

Las nuevas concepciones en Orientación se han reflejado en la terminología utilizada por lo que, actualmente, es frecuente la utilización de expresiones tales como Orientación para la Carrera y Asesoramiento para la Carrera; constituye, igualmente, una evolución reciente, la integración de la orientación en el cuadro del desarrollo personal y social, lo que es posible testimoniar en la afirmación «el asesoramiento para la carrera y el asesoramiento individual están inextricablemente relacionados. Los problemas de carrera tienen una fuerte componente emocional (...) es casi imposible separar o categorizar un problema como siendo de carrera o personal» (Krumboltz, 1993, p. 143). 
Sin embargo, las importantes repercusiones de los modelos referenciados, la investigación y la práctica, han mostrado una preocupación esencial con el adolescente y el joven adulto siendo, al revés, restricto el interés con relación a los acontecimientos posteriores a la entrada en el mundo del trabajo.

El surgimiento de nuevas realidades hace que se vuelva claro que personas de todas las edades pueden tener, en muchas circunstancias, necesidades y preocupaciones sobre la carrera y, además, que la sociedad puede beneficiar de programas y de servicios de asesoramiento para la carrera (Campbell y Heffernan, 1983), sobrepasándose, en definitivo, el enfoque inicial del comienzo del siglo XX, momento en el que la población objeto de estudio estaba confinada a los jóvenes que transitaban de la escuela hacia el mercado de trabajo.

De este modo, como objeto central de investigación y de intervención, el adulto recibe una creciente atención no solamente por parte de la comunidad científica y de los especialistas en asesoramiento sino también por parte de la sociedad, en general, y del mundo del trabajo, en particular. En orden a este cambio de actitudes prácticas y teóricas en mucho han concurrido las demandas del mercado de trabajo, en el sentido de proporcionar apoyo a personas de todas las edades y en todas las etapas de desarrollo (Johnston y Heppner, 1984; Law, 1981).

Entre los factores que han desencadenado la creciente atención hacia el adulto es legitimo que se refieran aquellos que reportan a los condicionantes de algunas profesiones, con el retorno de las mujeres con hijos ya mayores a la actividad profesional o bien con cambios de relieve de carácter social, económico o político; éstos constituyen factores considerados clásicos subrayándose, además, en la actualidad, otros de naturaleza demográfica, tecnológica o psicológica (Campbell y Heffernan, 1983; McCrae y Costa, 1990; Watts, 1980).

Los aspectos demográficos son particularmente importantes destacándose, entre ellos, el aumento de la esperanza de vida (por lo que hay que tener en cuenta la población con 65 años de edad, o más) y, además, el creciente número de mujeres incorporadas a la vida laboral, incluso las que poseen formación de nivel universitario (Campbell y Heffernan, 1983; Johnston y Heppner, 1984).

En lo que respecta a los datos de naturaleza tecnológica se hace clara la existencia de un creciente número de profesiones, muchas de las cuales no existían en el momento de la 
elección de carrera por parte de los adultos que actualmente se encuentran en el mercado de empleo; estos cambios ocurren, en gran medida, en áreas que recurren a las nuevas tecnologías siendo notable el hecho de que el patrón lineal escuela - trabajo - retiro ya no es compatible con los mismos, porque es más frecuente el cambio de tipo y de lugar de trabajo, a lo largo de la vida (Brown, 1998; Johnston y Heppner, 1984; Kerka, 1995).

Los cambios culturales y económicos en la sociedad y las modificaciones tecnológicas y organizacionales en los puestos de trabajo pueden generar frustración en un largo número de trabajadores. Además, alteraciones en los valores, necesidades y expectativas de los sujetos conducen en grande parte de los adultos a la reformulación de sus planes de carrera (Lankard, 1993).

Por lo expuesto, se asiste a una creciente flexibilidad en la carrera, aspecto traducible en las siguientes constataciones: la mediana edad no es ya, en lo que respecta a cuestiones profesionales, un período de acomodación y de mantenimiento del empleo; los fenómenos del desempleo y del subempleo han conducido al pensamiento de que la progresión en la carrera no se hace solamente en un sentido vertical sino también en un sentido transversal; las necesidades de formación continua conducen al imperativo de tomas de decisión de carrera consecuentes; el retiro anticipado, por cuestiones legales, implica la ponderación de actividades alternativas, de valor social; el surgimiento de nuevos estilos de vida induce el adulto a rehacer sus planes iniciales de carrera (Campbell y Heffernan, 1983; Neapolitan, 1980; Thomas, 1980; Watts, 1980). Así, son necesarias nuevas competencias y nuevas formulas de ajuste, surgiendo como un imperativo alcanzar una comprensión, integrando claramente el desarrollo personal de los sujetos, lo que es distinto de un enfoque de caracter estático.

Los aspectos referidos destacan, por una parte, la importancia del estudio del desarrollo del individuo, lo que se traduce en prácticas de asesoramiento pero, por otra parte, destacan una gran diversidad de poblaciones. En efecto, si es cierto que los psicólogos se han centrado tradicionalmente con preocupaciones de reingreso de las mujeres al mercado de trabajo o con hombres y mujeres que cuestionaban la dirección de su actual profesión y buscaban alternativas de carrera, en la actualidad se actúa sobre grupos distintos y específicos. Entre ellos se destacan: los trabajadores más viejos, los cuales buscan carreras después del retiro; los trabajadores desplazados, cuyos empleos han sido eliminados a causa de sucesos tales como el cierre de fincas, bancarrotas y fusiones de empresas; las parejas, en las cuales ambos 
trabajan y cuyas necesidades son más complejas que en aquéllas en las que solamente uno de los dos ejerce la carrera (Avedon, 1995; Goodman y Hoppin, 1988; Imel, 1996).

Los factores referenciados y las investigaciones sobre el adulto sirven como fundamentación para la necesidad de ayuda en el proceso de desarrollo de la carrera donde se puede poner el acento en distintos aspectos: los del desarrollo de la carrera, desde un punto de vista global, como un maxiciclo, con sus sucesivas etapas y los miniciclos que ocurren, a veces, en cada una de las etapas; puede valorar los distintos papeles a los que el sujeto es llamado a desempeñar, teniendo en cuenta su ponderación especifica en cada una de las etapas de vida y su recíproca interacción (particularmente importante en el adulto, en el cual pueden ocurrir conflictos entre los papeles familiar y profesional); puede, aún, volcarse hacia los "acontecimientos de la vida", es decir, ayudar al sujeto en el aprendizaje de la gestión de las transiciones ocasionadas por cambios previsibles o imprevisibles, de manera que pueda ejercer control, y no ser, controlado por ellas, y aprender a obtener de las mismas, ventajas en lo que respecta a oportunidades de crecimiento (Duarte, 2004a, 2004b; Watts, 1980).

\section{Una lectura de teorías: conceptualizaciones e intervenciones con adultos}

Aunque no específicamente direccionadas hacia la población adulta, las principales teorías desarrolladas en el contexto de la evolución de la Orientación pueden traducirse en contribuciones de relieve en lo que respecta a la comprensión de la dinámica del desarrollo del adulto y, además, suscitar pistas relevantes en el asesoramiento con adultos. Utilizando, siempre que sea apropiado, algunos de los criterios formulados por Krumboltz y Nichols (1990), se busca, en seguida, de entre los principales enfoques algunos de los aspectos que podrán enriquecer la comprensión del sujeto adulto.

Esta tarea implica una primera dificultad - ¿que modelos elegir? En realidad, las distintas clasificaciones de los modelos existentes se someten a distintos criterios de organización por lo que, en consecuencia, las clasificaciones son muy diversas. Veamos algunos ejemplos. Crites (1969) hace la distinción entre teorías no psicológicas (accidente, económicas, culturales y sociológicas) y teorías psicológicas (rasgo-factor, psicodinámicas, del desarrollo y de toma de decisión). Walsh (1990) subraya las teorías del rasgo-factor, las teorías centradas en la persona, las teorías psicodinámicas, las teorías del desarrollo, las teorías del aprendizaje social y la orientación asistida por ordenador. Osipow (1990) identifica un con- 
junto de cuatro teorías de relieve histórico y cultural: rasgo-factor, ajuste al trabajo, aprendizaje social y del desarrollo. Herr y Cramer (1996) hacen la distinción entre los enfoques del rasgo-factor, las teorías de la decisión, los enfoques situacionales, sociológicos y contextuales, los psicodinámicos y los del desarrollo. A su vez, Savickas y Lent (1994) destacan cinco autores como los más representativos de las actuales perspectivas en orientación: John D. Krumboltz, René V. Dawis, John L. Holland, Edward S. Bordin y Donald Super.

De los enfoques del rasgo-factor se extraen importantes aspectos en lo que respecta a la comprensión del adulto: las características personales, las características del ambiente y el proceso de ajuste. Resaltan, en la actualidad, los aportes de la teoría del ajuste al trabajo (theory of work adjustment) de Davis y Lofquist y la teoría de la personalidad vocacional y de los ambientes de trabajo (theory of vocational personalities and work environments) de Holland.

La teoría del ajuste al trabajo, al hacer la comparación entre las necesidades individuales y las demandas del ambiente con el objetivo de predecir el ajuste, la satisfacción y el mantenimiento del sujeto en las funciones que desempeña, operacionaliza los conceptos recurriendo a un conjunto de instrumentos de evaluación. Ofrece, así, una importante contribución en lo que respecta al diagnóstico de las características de personalidad del cliente (capacidades y necesidades psicológicas). Otra importante contribución consiste en hacer posible la evaluación de las características del ambiente permitiendo señalar las correspondencias y los desajustes significativos. De este modo, el psicólogo puede ayudar el cliente en las formas de ajuste siendo posible que el enfoque recaiga en el cambio del ambiente, del sujeto o de ambos, en el sentido de la búsqueda de la satisfacción del sujeto (grado de satisfacción en relación con el trabajo que desenvuelve o medida en la que las necesidades individuales se satisfacen por medio del trabajo) o de la organización (grado de satisfacción de la organización en relación con el desempeño del sujeto) (Dawis, 1994; Dawis y Lofquist, 1977; Lofquist y Dawis, 1991; Swanson, 1996).

A su vez, la teoría de Holland presenta un conjunto de ideas simple, partiendo de las mismas en dirección a elaboraciones más complejas. Las personalidades se caracterizan por su similitud con uno de seis tipos de personalidad (Realista, Intelectual, Artístico, Social, Emprendedor y Convencional); los ambientes en los cuales se desenvuelven las personas se caracterizan por su similitud con los seis modelos ambientales y con las mismas designaciones 
de los tipos de personalidad; por último, la combinación de personas y de ambientes se traduce en resultados predecibles y posibles de comprender tomando como punto de partida nuestro conocimiento de los tipos de personalidad y de los modelos ambientales. Así, al proponer un sistema comprensivo el cual permite una ligación explicita entre las características de personalidad y las características del ambiente ha permitido también, en resultado de amplios estudios empíricos, aportes al nivel de los instrumentos de evaluación de las variables personales, cuya utilidad se puede revelar en el proceso de diagnóstico (Holland, 1997).

En síntesis, una de las principales contribuciones de las perspectivas de rasgo-factor, según las especificaciones de cada uno de los autores, radica en el componente de la información, sea la información relacionada con el sujeto sea la referida al ambiente; a este propósito, es posible la visualización de la información como promotora del autoconocimiento (Lent, 1996). De referir, además, que las actuales perspectivas de rasgo-factor destacan la interacción recíproca entre las características individuales y las del ambiente, es decir, el individuo modela el ambiente y, a su vez, el ambiente modela el individuo (Swanson, 1996), aspecto que podrá poseer utilidad desde el punto de vista de la comprensión de los procesos de ajuste del adulto.

La posibilidad de establecer una imagen comprensiva del desarrollo de la carrera del individuo, constituye una notable contribución de las teorías del desarrollo en relación con la "representación de la realidad", al permitir localizar y ayudar a localizar al sujeto en un proceso dinámico de desarrollo. Las contribuciones de las distintas teorías difieren en cantidad, complejidad y especificidad de conceptos propuestos, y también en la dimensión de los estudios empíricos que las sostienen. Herr y Cramer (1996) subrayan, entre las teorías del desarrollo que abordan el adulto las de Super, Savickas y Schlosseberg.

Entre las teorías del desarrollo se destaca el "modelo del desarrollo, evaluación y asesoramiento para la carrera" (career-development asssessment counselling model) (Osborne, Brown, Niles, y Miner, 1997; Super, Osborne, Walsh, Brown, y Niles, 1992). Este es un modelo que deriva de un amplio conjunto de investigaciones realizadas por Donald Super, que considera que algunos de los aspectos del desarrollo de la carrera pueden ser evaluados e influenciados a través del recurso a la interpretación de los tests psicológicos y al asesoramiento para la carrera. Las teorizaciones en las cuales se basa el "modelo del desarrollo, evaluación y asesoramiento para la carrera" se agrupan en tres grandes categorías: "modelo del desarrollo 
de la carrera, madurez o adaptabilidad"; "modelo del arco iris de la carrera" y "modelo del arco normando de la carrera" (Osborne, Brown, Niles y Miner, 1997; Super, 1953, 1955, 1957, 1986, 1990) destacándose los siguientes aspectos:

- un componente diferencialista manifiesto en el significado atribuido a las características personales, a las diferencias individuales y a la interacción entre el individuo y el medio, en la aserción de la persona como agente activo en el proceso de desarrollo;

- una fuerte vertiente de desarrollo claramente enunciada en la formulación de una teoría en etapas y subetapas del desarrollo, caracterizadas por comportamientos vocacionales típicos (maxiciclo), por sobreposiciones posibles, por limites de edad flexibles, por transiciones igualmente flexibles y, aún, por reciclajes a lo largo de miniciclos;

- los conceptos de madurez y/o adaptabilidad y los respectivos modelos teóricos, con particular significado en el asesoramiento individual por su relieve en la prontitud del sujeto en la toma de decisión y en la interpretación de los tests psicológicos;

- la innovación conceptual, en la psicología de la carrera, resultante de la introducción del concepto de papeles (es decir, de la importancia relativa de los papeles), claramente expresados en el modelo del arco iris de la carrera; efectivamente, la carrera, observable a partir de una secuencia de etapas es, al igual, caracterizada por el desempeño de una variedad de papeles (roles) en un amplio universo de escenarios;

- la importancia de los distintos determinantes (biológicos, psicológicos y socioeconómicos) de las elecciones, integrados de forma comprensiva en el ciclo vital y en el espacio de vida y en su relación con los autoconceptos, que son de sobremanera evidentes en el modelo del arco normando de la carrera;

- la acentuación en el proceso de ayuda, a lo largo del ciclo vital (poniendo problemas de carrera y, además, en presencia de poblaciones distintas) y en diferentes contextos (académico, organizacional) concilia los nuevos modelos explicativos del funcionamiento psicológico en el adulto y las nuevas realidades (en la sociedad y en el mundo laboral) presentando formas de intervención flexibles. Así, se preconiza, en el modelo, un conjunto de instrumentos de evaluación de las dimensiones psicológicas más estrechamente relacionadas con las elecciones vocacionales. Se proponen distintas secuencias de utilización de esos mismos instrumentos, en una perspectiva flexible en las cuales la substitución o la adición de otros instrumentos permite evaluar dimensiones en función de necesidades de intervención o de investigación (Rafael, 2001; Rivas, 1998). 
A su vez, Schlossberg (1981) ha sintetizado el trabajo de otros teóricos del adulto de un modo pertinente en lo que respecta al desarrollo y al asesoramiento $\mathrm{y}$, al poner de manifiesto la importancia de los aspectos de naturaleza social en el contexto del comportamiento del adulto, los cuales poseen más relieve que los de carácter biológico (cuya ponderación es más visible en la infancia y en la adolescencia). Subraya, al mismo tiempo, las especificacones del adulto. El relieve atribuido por Schlossberg al concepto de transición (por opuesto a una visión de estabilidad y de certeza) sugiere al individuo adulto como una entidad que experimenta cambios, sea de modo deliberado sea en función de fuerzas que le son exteriores. Además, al caracterizar la transición en terminos de tipo, contexto e impacto $\mathrm{y}$, al defender que una transición debe ser examinada en función de los moldes en los que el sujeto hace la evaluación de la naturaleza de la transición, de los recursos existentes en el momento de la transición y de las características de la persona y del ambiente, aporta a la visión del adulto (y al asesoramiento) un "condimento" muy importante, el de la comprensión de la variabilidad y de su relevancia (Schlossberg, 1981).

La conceptualización de Savickas pone en relación el modo como las personas viven y estructuran el tiempo con sus concepciones de carrera y comportamientos vocacionales, defendiendo que el éxito y la satisfacción (en la carrera) se relacionan con las experiencias del tiempo (Herr y Cramer, 1996). Un importante aporte radica en la conceptualización de la experiencia del tiempo en el proceso de asesoramiento del adulto; se trata de una experiencia subjetiva del tiempo por oposición al tiempo objetivo como el que miden los relojes, el que deviene de la cronología, de la historia o de los calendarios (Ringle y Savickas, 1983).

La perspectiva del tiempo, la diferenciación del tiempo y la integración del tiempo son los factores integrados en esta concepción subjetiva del tiempo:

- la perspectiva del tiempo reporta la forma de como los sujetos (y las organizaciones) se visualizan y orientan en el tiempo; es determinada por la zona del tiempo (pasado, presente o futuro) que más relevancia asume en el proceso de decisión y en ella se incluyen imágenes, analogías y metáforas utilizadas por las personas con el objetivo de comprender el tiempo (Ringle y Savickas, 1983; Savickas, 1991);

- la diferenciación temporal se caracteriza por las dimensiones densidad y espacio. La densidad se relaciona con el número de sucesos que ocurren en un determinado período/zona del tiempo; por ejemplo, en lo que se refiere al futuro se verifica que las personas difieren 
claramente en el número de sucesos que suponen van a ocurrir en un período de cinco años en sus organizaciones/vidas; al igual, ejemplificando, relativamente al pasado, se puede constatar que algunos sujetos hacen relatos ricos de historia local mientras que otros conocen muy poco de lo sucedido antes de su ingreso en una institución. El espacio incluye aspectos de naturaleza retrospectiva y otros de naturaleza prospectiva; los primeros reportan en qué medida los sujetos son capaces de volver hacia atrás en la historia de su organización/vida mientras que los segundos se refieren a la extensión temporal en la cual se proyectan, o proyectan su organización en el futuro (Ringle y Savickas, 1983);

- la integración temporal respecto a la ligazón entre acontecimientos relativos a distintos períodos de tiempo contiene dos variables, la continuidad (de naturaleza cognitiva) y el optimismo (de naturaleza afectiva). Para estos autores, una dirección de continuidad facilita el establecimiento de relaciones interconectando el presente y el pasado y la relación del futuro con los actuales esfuerzos. Mientras que un sentido de discontinuidad del tiempo produce experiencias fragmentarias, desconectadas y frustrantes. El optimismo denota un sentido de confianza en el alcance de objetivos futuros (Ringle y Savickas, 1983).

En síntesis, para Savickas, las personas tienen necesidad de un rumbo objetivo de carrera que les permita poseer sentimientos conscientes sobre ellas mismas, integrando el pasado, el presente y el futuro. Una vez que la carrera subjetiva emerge de creencias y de actitudes hacia el tiempo, los conceptos de perspectiva del tiempo, de diferenciación y de integración temporal pueden ser utilizados en el proceso de asesoramiento con el objetivo de facilitar actitudes y actividades de planificación y favorecer positivamente los procesos de toma de decisión. Se trata de encuadrar, por medio de historias de vida, los objetivos personales (en un contexto significativo, anticipando sucesos, y de desarrollar actitudes y competencias de planificación, planes de contingencia y de acción direccionada que permita ayudar las personas a crear, articular y alcanzar sus aspiraciones (Marko y Savickas, 1998; Ringle y Savickas, 1983; Savickas, 1991).

Puede, por tanto, decirse que las teorías del desarrollo de la carrera a lo largo de la vida no constituyen un todo unitario, pero si una variedad de configuraciones, atribuyendo cada una de las mismas énfasis a distintos aspectos del trabajo, de los papeles de vida y del self (Law, 1998) contribuyendo, así, de manera diferente, hacia la comprensión del desarrollo del adulto. 
Con respecto a las perspectivas que se inscriben en el aprendizaje social se extrae, en primer plano, en el contexto del estudio del adulto, una explicación sobre como las personas hacen elecciones vocacionales, explicación que se basa en un conjunto de procesos de aprendizaje de naturaleza comportamental y cognitiva.

Efectivamente, al sugerir que las personas adquieren preferencias por distintas actividades por medio de experiencias de aprendizaje, se destacan como aspectos fundamentales la influencia del refuerzo en el desarrollo del autoconcepto y en la determinación del comportamiento; así, estos enfoques ponen el énfasis en el refuerzo de las respuestas deseables, en el modelaje y en la adquisición de competencias de toma de decisión (Walsh, 1990). Los procesos sociales y cognitivos pueden ser utilizados para explicar las diferencias individuales en la prontitud en el procesamiento y utilización de la información en el asesoramiento; el análisis del sistema de creencias, cogniciones, procesos metacognitivos en el asesoramiento, en conjunto con el análisis de la historia del trabajo, son importantes para la comprensión del individuo y para el dibujo de estrategias de intervención (Chartrand, 1996).

Concretando, la identificación de creencias que puedan estar influyendo en la capacidad del cliente para alcanzar objetivos, de mitos de carrera o actitudes ineficaces, en simultáneo con el análisis de los aprendizajes escolar y profesional, evidencian aspectos de utilidad que hay que tener en cuenta en el caso de adultos, para los cuales se vuelve necesario intervenir a nivel del aprendizaje, de la modificación, gestión y control del comportamiento (Krumboltz, 1991, 1994; Law, 1998).

Los enfoques dinámicos tienen como objetivo principal ayudar a las personas a descubrir oportunidades vocacionales socialmente viables y personalmente deseables, susceptibles de ayudarlas en el desarrollo de sus temas de vida y de apoyarlas en el desarrollo a través del trabajo. Así, señalan un proceso no solamente centrado en el ajuste vocacional, pero además en el ajuste general y personal poniendo en evidencia la comprensión del self, en un proceso cuya tendencia es la orientación terapéutica. Poniendo el enfoque en aspectos de naturaleza consciente e inconsciente, visualizan el sujeto como reactivo a impulsos, necesidades y motivos. Valoran la interpretación y la integración, en el contexto de la entrevista, de los resultados de los tests, como en el caso de las técnicas proyectivas, y de las autobiografías, como conductores de un diálogo hacia la comprensión y la autocomprensión del sujeto (Walsh, 1990). 
El modelo psicodinámico de la elección y de la satisfacción en la carrera de Bordin destaca la importancia de la personalidad en el desarrollo y en las decisiones vocacionales, integrando conceptos llave tales como la importancia del juego, de la espontaneidad, de la compulsión y del esfuerzo. Entre las implicaciones del modelo, la consideración del sujeto como un todo y no solamente en su papel de trabajador asume especial relieve; de este modo, se destaca, además de una consideración de las realidades del trabajo, una particular atención a los afectos. De este modo, el análisis de toda la historia del sujeto es ampliamente valorada en el proceso de asesoramiento en orden a intentar comprender de qué modo el sujeto se visualiza y qué conflictos experimenta proponiéndose, como posibles estrategias, la realización de ejercicios de fantasía dirigida con el objetivo de liberar el sujeto de los constreñimientos de la realidad y de conceder libertad a su vida interior (Bordin, 1990, 1994). Los aspectos mencionados a propósito de los modelos dinámicos, aunque puedan ser considerados en procesos de intervención suscitan sin embargo, dificultades de operacionalización y, en consecuencia, de aplicabilidad y de verificación científica.

\section{Desarrollo y gestión de carreras en el siglo XXI: en búsqueda de nuevas lecturas}

Con anterioridad hemos referido que el incremento de la atención hacia el adulto, reflejada en la multiplicidad de poblaciones destinatarias de la intervención y de las áreas y contenidos de los programas de intervención, se ha traducido en una clara ruptura con las concepciones del comienzo del siglo XX, poniendo el acento en un enfoque del desarrollo de la carrera a lo largo de la vida en el que los aspectos de naturaleza personal asumen una importancia central. Tomando las palabras de Brown y Brooks (1991, p.5) «el asesoramiento para la carrera es un proceso integrado destinado a ayudar a los sujetos con problemas de desarrollo de la carrera (...) es un proceso de elección, ingreso, ajuste y avance en una profesión (...) es un proceso que interactúa de forma dinámica con otros papeles en la vida» por lo que «los problemas de carrera incluyen, pero no se limitan, a la decisión e indecisión en la carrera, al desempeño en el trabajo, al estrés y al ajuste, a la incongruencia del sujeto y del ambiente de trabajo y a la inadecuada o insatisfactoria integración de los papeles».

Hemos, también, referido anteriormente algunas teorías cuyas contribuciones hacia la comprensión del adulto y la fundamentación de la intervención se asumen como relevantes. La creciente atención hacia el adulto se refleja también en las recientes revisiones de la litera- 
tura (Guindon y Richmond, 2005) destacando específicamente la necesidad de incrementar la intervención en el mundo del trabajo (Watts, 2005).

En la evolución de las perspectivas sobre el adulto emerge también la importancia de la responsabilidad del sujeto en la gestión de su carrera apoyada, a menudo, por las organizaciones a través de los programas que ponen a disposición de sus trabajadores, los cuales pueden asumir una gran diversidad de formas (Arnold, 1997; Gottfredson, 2005; Kidd, 1998).

Así, es posible asistir a una atención muy particular en lo que se refiere a la inserción del adulto en el mundo del trabajo aspecto que, en algunos casos, conduce a un análisis de la interacción del sujeto con la organización y, en consecuencia, al establecimiento de estrechas relaciones entre la carrera individual y las demandas de la organización, las cuales circunscriben etapas, posiciones y procesos psicológicos a los cuales los psicólogos deberán estar atentos.

Sin embargo, el mundo del trabajo - un escenario privilegiado en el cual se mueve el adulto - sufre en la actualidad profundas modificaciones señaladas por múltiples autores de diferentes áreas del conocimiento. Nos hallamos, indubitablemente, en una sociedad del conocimiento caracterizada por la globalización, internacionalización y tecnología en la que el cambio aparenta ser la única certeza, no solamente para los sujetos como también para las organizaciones. Cambios en las organizaciones y en el trabajo (e.g. fusiones; competitividad y presión hacia la productividad; diversidad étnica y cultural), pero también cambios en las oportunidades y desafíos presentados a los sujetos (e.g. naturaleza de los contratos laborales; creciente énfasis en competencias de caracter tecnológico e interpersonal - teamwork, networking; desempleo; crecientes oportunidades de trabajo en distintas partes del mundo; necesidad de considerar opciones de autoempleo; patrones de carrera menos estables y predictibles; menor oportunidad de promoción vertical; conciliación entre el trabajo y la familia) (Amundson, 2005; Baruch, 2004; Greenhaus, Callanan, y Godshalh, 2000).

Estas nuevas realidades obligan a replantear y revisar las perspectivas de que disponemos y a encontrar nuevas respuestas a los retos con los las que personas, las organizaciones y la sociedad se enfrentan. No se trata de pretender encontrar, aquí, respuestas (u orientar tendencias) pero solamente indicar unos cuantos puntos de vista que la "anciana" Orientación, mejor dicho, el Desarrollo y Gestión de Carreras, expresión más adecuada desde nuestro pun- 
to de vista, deberá incluir bajo el riesgo de echar a perder su impacto (históricamente incuestionable) en la vida de los sujetos y de las organizaciones.

Se trata de responder a lo Global/Internacional, de responder a lo Organizacional, y de responder a lo Individual y, en cada uno de esos niveles, reflejar los papeles de la teoría, de la investigación y de la intervención. Veamos, sin una preocupación exhaustiva, algunos de esos desafíos. Evidentemente, constituyen tareas y desafíos de cada uno de los profesionales pero son, esencialmente, desafíos de un área que se denomina: Desarrollo y Gestión de Carreras.

\section{Responder a la Globalización /Internacionalización}

El mundo es, en un plano global, uno de los escenarios de las organizaciones y de las personas. La Psicología del Desarrollo y Gestión de Carreras (con adultos) tiene, en este nivel, importantes retos, algunos de los cuales se han hecho visibles a lo largo del análisis de su historia.

Es el caso del número sustancial de instrumentos y técnicas de evaluación, muchas de las cuales adaptadas a otros países y, al revés de lo sucedido hasta hace poco tiempo, fácilmente accesibles al público a través de Internet. Como señala Whiston (2003), uno de los retos se refiere a la garantía de que los instrumentos colocados a disposición dispongan de sólidas bases psicométricas. Posiblemente, una de las respuestas pasará por la influencia en la reglamentación de los servicios proporcionados a través de Internet, aspecto en el que las asociaciones científicas y profesionales de esta área deberán desempeñar un papel central.

La respuesta, a un nivel global, pasa también por el incremento de las redes de colaboración internacionales sea en proyectos de investigación ${ }^{1}$, o de formación, sea al nivel de la edición y de la divulgación (e.g., revistas de carácter verdaderamente internacional).

Más específicamente, pasará también por la atención a nuevos fenómenos de internacionalización de las carreras (en particular, en un futuro cercano, al nivel de los cuadros superiores) y de la diversidad cultural, étnica y lingüística, fenómeno que empieza a ganar visibili-

\footnotetext{
1 Un excelente ejemplo de este tipo de trabajo es el Estudio de la Importancia del Trabajo - Work Importance Study (Super y Šverko, 1995).
} 
dad en Europa al nivel de la movilidad de estudiantes, profesores e investigadores como consecuencia de la Declaración de Bolonia.

La respuesta pasará también por revitalizar la importancia de la cultura, mejor dicho, de las culturas, sea en el impacto sobre los sujetos, sea en el impacto sobre las organizaciones (en la línea de los trabajos pioneros de Geert Hofstede, 1991), si se considera la probable expansión del Desarrollo y Gestión de Carreras a grupos menos tradicionales y a países que empiezan, en la actualidad, a desarrollar actividades en este ámbito, como refiere Pope (2003). Estamos, así, delante de la inevitabilidad de la consideración de las repercusiones de la multiculturalidad y de la interculturalidad.

Naturalmente, muchos de estos retos se ponen a los que, en el dominio del Desarrollo y Gestión de Carreras, acceden e influyen en niveles de fuerte intervención, designadamente en asociaciones y organismos internacionales.

\section{Responder a la organización}

Las presiones generadas por las imposiciones de productividad plantean, como ya hemos dicho, nuevos retos. Una consecuencia directa obliga a que las intervenciones en el contexto del Desarrollo y Gestión de Carreras adopten, sin pérdida de rigor científico, algún pragmatismo y, como señala muy acertadamente Niles (2003) las intervenciones deberán ser documentadas y mostrar claramente que los servicios son eficaces. Se trata, a menudo, de transformar lo que es académico y teórico en un lenguaje de carácter más empresarial y, en consecuencia, más útil desde el punto de vista de las intervenciones organizacionales. Este componente exige una actitud más emprendedora, algo que la mayoría de los psicólogos no siempre están particularmente atentos; dado que su intervención se enfoca, tradicionalmente, en el individuo.

Como temas más específicos, permanecen también actuales (en agenda) las contribuciones hacia el planeamiento y la integración de los recursos humanos (e.g. procesos de acogimiento, reclutamiento y selección de personal; gestión y evaluación del desempeño; formación). Las nuevas realidades de las empresas empujan hacia la diversificación del dominio tradicional del asesoramiento para la carrera y a la integración de la gestión de carreras en los sistemas de recursos humanos, designadamente a través de intervenciones organizacionales de los que son ejemplos el acompañamiento (coaching), la tutoría y el outplacement. Algunas de 
las cuestiones asociadas son igualmente merecedoras de atención: véase, por ejemplo, el ajustadísimo comentario de Whiston (2003) que, al referir la actual popularidad de los se titulan coach, los cuales ganan elevadas remuneraciones pero cuya preparación es deficitaria, refiere también el hecho de constituir una amenaza directa a los que se encuentran debidamente preparados y poseen las competencias y los conocimientos adecuados para efectuar intervenciones eficaces.

Naturalmente, muchos de los temas de caracter organizacional no se encuentran, ni es supuesto que estén, bajo la exclusividad de profesionales del Desarrollo y Gestión de Carreras. Estos temas son, en gran medida, además de áreas científicas, objetos de análisis o problemas de intervención necesariamente multidisciplinares. En este punto radica otro importante desafío para los psicólogos consistiendo en considerar las contribuciones de otras áreas del conocimiento (e.g. economía, derecho, sociología) en el sentido de posibilitar una respuesta efectiva a las necesidades de las organizaciones y hallar lenguajes y metodologías de entendimiento interdisciplinar.

\section{Responder al individuo}

Finalmente, nos referimos al pimer nivel histórico y más tradicional: el del individuo. Sin olvidar el papel de las teorías existentes, como se ha referido anteriormente, se destaca, en la actualidad, la importancia de los nuevos enfoques constructivistas y contextuales (Guichard y Lenz, 2005; Richardson, 2002, 2004; Savickas, 2005) y su relieve como marcos de referencia en la investigación y la intervención.

Sin embargo, no es posible olvidar que la respuesta al individuo pasa indubitablemente por responder, también, a las nuevas cuestiones que le depara. Constituyen ejemplos de temas críticos: la particular atención al aprendizaje a lo largo de la vida; el poner en primer plano la "empleabilidad" y no el empleo en las intervenciones individuales; la calidad de vida y la calidad de vida en el trabajo, el estrés profesional, la satisfacción profesional; los planes de carrera y de sucesión; el desempleo; el envejecimiento y el envejecimiento activo; la gestión de la diversidad; el tiempo libre; la satisfacción y productividad.

Independientemente del nivel considerado, las respuestas del Desarrollo y Gestión de Carreras exigen interdisciplinaridad, multidisciplinaridad y contextualización. 


\section{Conclusiones: lecturas finales}

En la historia aún relativamente reciente de la Orientación, como problema científico, los enfoques del desarrollo de la carrera a lo largo de la vida han emergido en la década de los 50 del siglo XX, contraponiéndose a las concepciones de Parsons vigentes hasta entonces. Sin embargo, si las concepciones del desarrollo han empezado a dirigir la práctica de la orientación, la población de adultos solo ha recibido, en un pasado reciente, una acrecida atención por parte de la comunidad científica y de la sociedad. Esta atención surge en consecuencia de un conjunto de factores de naturaleza demográfica, tecnológica y psicológica, que ha sobrevalorado la importancia de los cambios que ocurren en el sujeto adulto en la sociedad. Efectivamente, cambio es la palabra clave: cambio en el mundo del trabajo, cambio en la mirada personal sobre la importancia del trabajo, cambio en las necesidades, en los valores y en las expectativas de los sujetos acrecidas de la evolución en los modelos teóricos.

Pero la naturaleza del trabajo es ahora distinta de lo que era en el pasado (y, además, en un pasado todavía reciente) siendo los fenómenos de la competitividad global, de la diversidad cultural, de las nuevas tecnologías y de los nuevos procedimientos de gestión realidades que requieren sujetos con un pensamiento critico, competencias en la resolución de problemas y de comunicación, al igual que elevados niveles de competencia profesional (Lankard, 1996). A su vez, el significado del trabajo es variable según el sujeto (Ferreira Marques, 1998; Herriot, 2001) y en el mismo sujeto a lo largo del tiempo (Herriot, 2001).

Estas realidades suscitan naturalmente la necesidad de una creciente y sistemática colaboración entre los teóricos del Desarrollo y Gestión de Carreras, los prácticos y los investigadores, como miembros de equipos de investigación, hacia la comprensión del comportamiento vocacional en contextos específicos y con poblaciones, también ellas, específicas (Herr y Cramer, 1996).

Así, es improbable que una sola teoría sea adecuada para explicar y fundamentar las intervenciones en el Desarrollo y Gestión de Carreras. Sin embargo, este hecho no constituye, desde nuestro punto de vista, un problema. Efectivamente, como sugiere Gergen (2001), al reflejar sobre la proliferación de metodologías y de paradigmas, si las teorías científicas y las metodologías solamente proporcionan una visión incompleta de la realidad, entonces la adop- 
ción de múltiples perspectivas teóricas y metodologías conducirá a un enriquecimiento en la comprensión de los fenómenos psicológicos.

La Psicología del Desarrollo y Gestión de Carreras ha acompañado a profundos cambios en la sociedad y en el concepto de trabajo, desde el final de la sociedad industrial hasta las épocas de la información y del conocimiento. En este trayecto, más que sobrevivir, se ha revitalizado, se ha adaptado, $\mathrm{y}$ ha propuesto he intervenido utilizando sólidas fundamentaciones teóricas y pruebas generadas en el terreno. Tomando en consideración ese fértil pasado de éxito como un predictor del futuro, seguramente se encuentra capacitada para, en la actualidad, responder a los nuevos retos que se le colocan. Sin embargo, esos desafíos se sitúan, en el presente, en nuevos niveles; más allá del individual (el nivel tradicional, pero aún central), pasando por el organizacional pero, sobretodo, en el comienzo del siglo, por la emergencia del nivel global.

La búsqueda de respuestas en los niveles considerados - respuestas que, en ultima instancia, constituirán la propia identidad del Desarrollo y Gestión de Carreras - pasa, inequívocamente por la teorización, investigación e intervención.

Del pasado se retiran seguramente indicadores y enseñanzas esenciales como hemos visto a través de las lecturas que sobre éste hemos hecho. Sin embargo, la complejidad de los actuales retos impone pensar (pensar global, pensar organizacional y pensar individual) en la conceptualización de la teoría y de la investigación como soportes basilares de la intervención. Las respuestas no son únicas ni lineales pero si "plurilineales" y "interlineales" dado el inevitable cruce de los niveles considerados. Pensar, y solamente a título de ejemplo, en el Individuo/Individuo (e.g., en el asesoramiento para la carrera) es seguramente distinto de pensar en el Individuo/Organización (e.g., en reclutamiento y selección de personal) o en el Individuo/Globalización (e.g., los sistemas y planes de carrera internacionales). Según los niveles, cruces de niveles resultantes, y direccionalidad, se exige también una creciente atención relativamente a otras áreas.

Intervenir en Psicología no puede ser, por tanto, intervenir tan sólo en Psicología, o pensar Psicología, sino también pensar político, pensar económico, pensar social y pensar cultural, es decir, Pensar Interdisciplinar y Pensar Pluridisciplinar, es decir, en cada momento (de la teoría, de la investigación y de la práctica) la necesidad de nuevas lecturas y de nue- 
vas respuestas a los desafíos buscando siempre la armonización de lo global y de lo individual.

\section{Referencias}

Amundson, N. (2005, September). Challenges for career interventions in changing contexts. Paper presented at the International Association of Educational and Vocational Guidance International Conference, Lisbon, Portugal.

Arnold, J. (1997). Managing careers into the 21st century. London: Paul Chapman Publishing Company.

Avedon, L. (1995). Older workers in transition. ERIC Digest. Columbus: ERIC Clearinghouse on Adult, Career and Vocational Education (ED400474).

Baruch, Y. (2004). Managing careers. London: Prentice-Hall.

Bordin, E. S. (1990). Psychodynamic model of career choice and satisfaction. En D. Brown y L. Brooks (Eds.), Career choice and development ( $2^{\text {nd }}$ ed.) (pp. 102-144). San Francisco: Jossey Bass.

Bordin, E. S. (1994). Intrinsic motivation and the active self: convergence from a psychodynamic perspective. En M. Savickas y R. Lent, Convergence in career development theories: implications for science and practice (pp. 53-61). Palo Alto: Consulting Psychologists Press.

Brown, B. L. (1998). Career mobility. A choice or necessity? Columbus: ERIC Clearinghouse on Adult, Career and Vocational Education (ED414436).

Brown, D. y Brooks, L. (1991). Career counseling techniques. Boston: Allyn \& Bacon.

Campbell, R. E., y Heffernan, J. M. (1983). Adult vocational behavior. En W. B. Walsh y S. H. Osipow (Eds.), Handbook of Vocational Psychology: Vol. 1. Foundations (pp. 223260). New Jersey: Lawrence Erlbaum Associates.

Chartrand, J. M. (1996). Linking theory with practice. A Sociocognitive interactional model for career counseling. En M. L. Savickas y W. B. Walsh (Eds.), Handbook of career counseling. Theory and practice (pp. 121-134). Palo Alto: Davies-Black Publishing.

Crites, J. O. (1969). Vocational psychology. The study of vocational behavior and development. New York: McGraw-Hill.

Crites, J. O. (1981). Career counseling. Models, methods and materials. New York: McGrawHill Book Company. 
Dawis, R. V. (1994). The theory of work adjustment as convergent theory. In M. Savickas, y R. Lent, Convergence in career development theories: implications for science and practice (pp. 33-43). Palo Alto: Consulting Psychologists Press.

Dawis, R. V. y Lofquist, L. H. (1977). Personality style and the process of work adjustment. En H. J. Peters y J. C. Hansen (Eds.), Vocational guidance and career developmentselected readings ( $3^{\text {rd }}$ ed., pp.329-335). New York: McMillan Publishing Co. Inc.

Duarte, M. (2004a, August). The gap between employees' careers and managers' policies: guilties and not guilties. Paper presented at the $28^{\text {th }}$ International Congress of Psychology, Beijing, China.

Duarte, M. E. (2004b). O indivíduo e a organização: perspectivas de desenvolvimento. Psychologica (Extra-Série), 549-557.

Ferreira Marques, J. H. (1993). El compromiso de la orientación escolar y profesional con los cambios de la sociedad. In V Seminario Iberoamericano de Orientación (pp.67-75), Tenerife: Asociación Española para la Orientación Escolar y Profesional.

Ferreira Marques, J. H. (1998). Challenges for guidance in the world. En E. R. Repetto Talavera, y C. Vélaz de Medrano (Eds.), Career development, human resources and labour market (pp. 393-403). Madrid: Universidad Nacional de Educación a Distancia., Vol. II.

Gergen, K. J. (2001). Psychological science in a postmodern context. American Psychologist, $56(10), 803-813$

Ginzberg, E. (1952). Toward a theory of occupational choice. The Personnel and Guidance Journal, 30 (8), 491-494.

Goodman, J., y Hoppin, J. (1988). Adult career counseling - new clienteles. Highlights: An ERIC/CAPS Digest. Columbus: ERIC Clearinghouse on Counseling and Personnel Services (ED3044624).

Gottfredson, G. D. (2005). Career development in organizations. En B. Walsh y M. Savickas (Eds.), Handbook of vocational psychology ( $3^{\text {rd }}$ ed., pp. 297-318). Mahwah, NJ: Lawrence Erlbaum Associates.

Greenhaus, J., Callanan, G., y Godshalh, V. (2000). Career management ( $^{\text {rd }}$ ed.). Mason, $\mathrm{OH}$ : Thompson.

Guichard, J. y Lenz, J. (2005). Career theory from an international perspective. The Career Development Quarterly, 54, 17-28.

Guindon, M. H., y Richmond, L. J. (2005). Practice and research in career counseling and development - 2004. The Career Development Quarterly, 54, 90-137. 
Hackett, G., Lent, R. W., y Greenhaus, J. H. (1991). Advances in vocational theory and research: A 20-year retrospective. Journal of Vocational Behavior, 38, 3-38.

Herr, E. L., y Cramer, S. H. (1996). Career guidance and counseling through the life span $\left(5^{\text {th }}\right.$ ed.). New York: Harper Collins.

Herriot, P. (2001). The employment relationship. A psychological perspective. Philadelphia: Routledge Ltd.

Hofstede, G. (1991). Cultures and organizations, software of the mind. Intercultural cooperation and its importance for survival. London: HarperCollinsBusiness.

Holland, J. L. (1997). Making vocational choices. A theory of vocational personalities and work environments ( $3^{\text {rd }}$ ed.). Odessa: Psychological Assessment Resources.

Hopson, B., y Scally, M. (1980). Change and development in adult life: Some implications for helpers. British Journal of Guidance and Counselling, 8 (2), 175-187.

Imel, S. (1996). Myths and realities: Older workers. Columbus: ERIC Clearinghouse on Adult, Career and Vocational Education (ED392894).

Jepsen, D. A. (1990). Developmental career counseling. En B. Walsh, y S.H. Osipow (Eds.), Career counseling: Contemporary topics in vocational psychology (pp. 117-157). New Jersey: Lawrence Erlbaum Associates.

Johnston, J. A., y Heppner, M. J. (1984). Adult career development: an overview. Highlights: An ERIC/CAPS fact sheet. Columbus: ERIC Clearinghouse on Counseling and Personnel Services (ED260370).

Kerka, S. (1995). Adult career counseling in a new age. Columbus: ERIC Clearinghouse on Adult, Career and Vocational Education (ED389881).

Kidd, J. M. (1998). Career planning within work organisations. En A. G. Watts, B. Law, J. Killeen, J. M. Kidd, y R. Hawthorn (Eds), Rethinking career education and guidance: theory, policy and practice (pp.142-154). London and New York: Routledge.

Krumboltz, J. D. (1991). Manual for the Career Beliefs Inventory. Palo Alto: Consulting Psychologists Press.

Krumboltz, J. D. (1993). Integrating career and personal counseling. The Career Development Quarterly, 42 (2), 143-148.

Krumboltz, J. D. (1994). Improving career development theory from a social learning perspective. En M. Savickas, y R. Lent, Convergence in career development theories: implications for science and practice (pp. 9-31). Palo Alto: Consulting Psychologists Press. 
Krumboltz, J. D., y Nichols, C. W. (1990). Integrating the social learning theory of career decision making. En B. Walsh y S. Osipow (Eds.), Contemporary topics in vocational psychology (pp. 159-192). New Jersey: Lawrence Erbaum Publishers.

Lankard, B. A. (1993). Career development through self-renewal. ERIC Digest. ERIC Clearinghouse on Adult, Career and Vocational Education (ED358378).

Lankard, B. A. (1996). Job training versus career development: What is voc ed's role? ERIC Clearinghouse on Adult, Career and Vocational Education (ED395217).

Law, B. (1981). Community interaction: A 'Mid-Range' focus for theories of career development in young adults. British Journal of Guidance and Counseling, 9 (2), pp. 142 158.

Law, B. (1998). A career-learning theory. En A. G. Watts, B. Law, J. Killeen, J. M. Kidd, y R. Hawthorn (Eds), Rethinking career education and guidance: theory, policy and practice (pp. 46-71). London and New York: Routledge.

Lent, E. B. (1996). The person focus in career theory and practice. En M. L. Savickas y W. B. Walsh (Eds.). Handbook of career counseling. Theory and practice (pp. 109-120). Palo Alto: Davies-Black Publishing.

Lofquist, L. H., y Dawis, R. V. (1991). Essentials of person environment correspondence counseling. Minneapolis: University of Minnesota Press.

Marko, K. W., y Savickas, M. L. (1998). Effectiveness of career time perspective intervention. Journal of Vocational Behavior, 52, 106-119.

McCrae, R. R., y Costa, P. T. Jr. (1990). Personality in adulthood. New York: Guilford Press.

Neapolitan, J. (1980). Occupational change in mid-career: An exploratory investigation. Journal of Vocational Behavior, 16, 212-225.

Niles, S. G. (2003). Career counselors confront a critical crossroad: a vision of the future. The Career Development Quarterly, 52, 70-77.

Osborne, W. L., Brown, S., Niles, S., y Miner, C. U. (1997). Career development, assessment and counseling: Applications of the Donald E. Super C-DAC model. Alexandria: American Counseling Association.

Osipow, S. H. (1990). Convergence in theories of career choice and development. Journal of Vocational Behavior, 36, 122-131.

Parsons, F. (1909). Choosing a vocation. Boston: Houghton Mifflin.

Pope, M. (2003). Career counseling in the twenty-first century: Beyond cultural encapsulation. The Career Development Quarterly, 52, 54-60. 
Rafael, M. (2001). O modelo desenvolvimentista de avaliação e aconselhamento de carreira (C-DAC). Preocupações de carreira, crenças de carreira e stress profissional em adultos trabalhadores. Dissertação de doutoramento em Psicologia da Orientação Escolar e Profissional. Lisboa: Faculdade de Psicologia e de Ciências da Educação.

Richardson, M. S. (2002). A metaperspective for counseling practice: A response to the challenge of contextualism. Journal of Vocational Behavior, 61, 407-423.

Richardson, M. S. (2004). The emergence of new intentions in subjective experience: A social/personal constructionist and relational understanding. Journal of Vocational Behavior, 64, 485-498.

Ringle, P. M., y Savickas, M. L. (1983). Administrative leadership. Planning and time perspective. Journal of Higher Education, 54 (6), pp. 649-661.

Rivas, F. (1998). Psicología vocacional: enfoques del asesoramiento. Madrid: Ediciones Morata.

Rogers, C. (1942). Counseling and psychotherapy: Newer concepts in practice. Boston: Houghton Mifflin.

Savickas, M. L. (1991, August). Career time perspective: Turning a model into an intervention. Paper presented at the Symposium "Career development research: turning theoretical constructs into counseling interventions", conducted at the 99th annual convention of the American Psychological Association, San Francisco.

Savickas, M. (2005). The history of vocational psychology. En B. Walsh y M. Savickas (Eds.). Handbook of vocational psychology ( ${ }^{\text {rd }}$ ed., pp. 15-50). Mahwah, NJ: Lawrence Erlbaum Associates.

Savickas, M. L., y Lent, R. W. (1994). Introduction. A convergence project for career psychology. En M. L. Savickas, y R. W. Lent, Convergence in career development theories: implications for science and practice (pp.1-6). Palo Alto: Consulting Psychologists Press.

Scholssberg, N. K. (1981). A model for analysing human adaptation to transition. The Counseling Psychologist, 9 (2), 2-18.

Super, D. E. (1953). A theory of vocational development. American Psychologist, 8 (4),185190.

Super, D. E. (1955). From vocational guidance to counseling psychology. Journal of Counseling Psychology, 2, 3-9.

Super, D. E. (1957). The psychology of careers. An introduction to vocational development. New York: Harper y Brothers. 
Super, D. E. (1986). Life career roles: self-realization in work and leisure. En D. Hall y Ass. (Eds.). Career development in organizations (pp. 95-119). San Francisco: JosseyBass.

Super, D. E. (1990). A life-span, life-space approach to career development. In D. Brown, y L. Brooks (Eds.). Career choice and development (2 ${ }^{\text {nd }}$ ed., pp. 197-261). San Francisco: Jossey-Bass.

Super, D. E., Osborne, W. L., Walsh, D. J., Brown, S. D., e Niles, S. G. (1992). Developmental career assessment and counseling: The C-DAC model. Journal of Counseling \& Development, 71, 74-79.

Super, D. E. y Šverko, D. (1995) (Eds.) Life roles, values and careers: International findings of the Work Importance Study. San Francisco: Jossey-Bass.

Swanson, J. L. (1996). The theory is the practice. Trait-and-factor/person environment fit counseling. En M. L. Savickas y W. B. Walsh (Eds.), Handbook of career counseling. Theory and practice (pp. 93-108). Palo Alto: Davies-Black Publishing.

Thomas, E. L. (1980). A typology of mid-life career changers. Journal of Vocational Behavior, 16, 173-182.

Vasconcelos, F. (1936). A escolha da carreira para os nossos filhos. Lisboa: Livraria Clássica Editora.

Walsh, W. B. (1990). A summary and integration of career counseling approaches. En B. Walsh y S. Osipow (Eds.), Contemporary topics in vocational psychology (pp. 263282). New Jersey: Lawrence Erlbaum Publishers.

Watts, A. G. (1980). Educational and careers guidance services for adults: A rationale and conceptual framework. British Journal of Guidance and Counselling, 8 (1), 11-22.

Watts, A. G. (2005). Career guidance policy: An international perspective. The Career Development Quarterly, 54, 66-76.

Whiston, S. C. (2003). Career counseling: 90 years old yet still healthy and vital. The Career Development Quarterly, 52, 35-42. 
Manuel Joaquim Enriques Rafael

[Página en blanco por razones de paginación] 